\title{
Short communication: An inducible CRISPR/dCas9 gene repression system in Lactococcus lactis
}

\author{
Zhi-Qiang Xiong, ${ }^{1}$ (1) Yun-Ying Wei, ${ }^{1}$ Ling-Hui Kong, ${ }^{1}$ Xin Song, ${ }^{1}$ Hua-Xi Yi, ${ }^{2} \odot$ and Lian-Zhong Ai ${ }^{1 *}$ (1) \\ ${ }^{1}$ Shanghai Engineering Research Center of Food Microbiology, School of Medical Instrument and Food Engineering, \\ University of Shanghai for Science and Technology, Shanghai 200093, China \\ ${ }^{2}$ College of Food Science and Engineering, Ocean University of China, Qingdao 266003, China
}

\section{ABSTRACT}

Lactococcus lactis, one of the most important probiotic lactic acid bacteria (LAB), is widely used in the dairy industry as a cell factory for recombinant protein production. Currently, a nisin-controlled inducible expression system is used for this purpose and represents the only commercial expression system in LAB. However, the available genetic modification methods are rather limited for modulating gene expression in L. lactis. Here, we developed a 2-plasmid system for gene transcription repression in L. lactis NZ9000 that uses inducible clustered regularly interspaced short palindromic repeats (CRISPR)-dCas9. An inducible promoter $\mathrm{P}_{\text {nisin }}$ was used to drive the expression of dCas9 from Streptococcus pyogenes, whereas a strong constitutive promoter $\mathrm{P}_{44}$ drove single guide RNA expression for single or multiple target genes. dCas9 enabled CRISPR interference-mediated silencing of single or multiple target genes with significant reduction of gene expression, up to $99 \%$. In addition, LLNZ_07335, a putative penicillin acylase, was identified as bile salt hydrolase for bile salt resistance in NZ9000 using this system. To our knowledge, this report is the first for a functional gene for bile salt tolerance in L. lactis. Overall, our work introduces a new gene repression tool for various applications in L. lactis or other LAB.

Key words: Lactococcus lactis, CRISPR interference, inducible dCas9, gene repression

\section{Short Communication}

Lactococcus lactis, a food-grade lactic acid bacteria $(\mathbf{L A B})$, is widely applied in the dairy industry and also used as a cell factory for recombinant protein production. Because of its lack of endotoxins and being generally recognized as safe, L. lactis has been developed as a

Received July 28, 2019.

Accepted August 30, 2019.

*Corresponding author: ailianzhong@hotmail.com vehicle for delivering antigens and therapeutic proteins for human health through genetic engineering (Berlec et al., 2018). Various tools have been relatively well developed for the expression of recombinant protein in L. lactis compared with other LAB. Among these tools, the nisin-controlled inducible expression (NICE) system is the most common tool for protein expression in L. lactis. Nevertheless, the toolbox available for L. lactis cannot compare with other well-established bacterial systems such as Escherichia coli. Hence, new genetic manipulation tools are highly desirable for use in L. lactis.

As a third-generation gene scissor, clustered regularly interspaced short palindromic repeats (CRISPR)-Cas9 is a powerful tool for synthetic biology and genome engineering in various eukaryotic and prokaryotic organisms. Various CRISPR-based technologies that offer precision gene editing have opened new avenues for unraveling the probiotic function of LAB and developing next-generation probiotics (Hidalgo-Cantabrana et al., 2017). Among LAB, species in the genus Lactobacillus have developed their own CRISPR-Cas9 systems for efficient genome editing (Oh and van Pijkeren, 2014; Song et al., 2017; Huang et al., 2019). For example, our group constructed CRISPR-Cas $9^{\mathrm{D} 10 \mathrm{~A}}$ nickase-based genome editing in $L$. case $i$ with efficiencies of 25 to $62 \%$ for in-frame deletions and chromosomal insertion (Song et al., 2017). Based on the NICE system, Berlec et al. (2018) recently constructed an inducible dual promoter plasmid to drive Cas9/mutated, nuclease-inactivated Cas9 (dead Cas9 or dCas9) and single guide RNA (sgRNA). Co-expression of sgRNA and Cas9/dCas9 can induce a double-strand break or transcriptional repression of a target gene (Berlec et al., 2018), demonstrating that the CRISPR-Cas9 system has application potential in L. lactis; however, genome editing (e.g., deletion and insertion) and multiple gene repression were not available.

To achieve multiple gene repression in L. lactis, we constructed an inducible CRISPR-dCas9 (also called CRISPR interference, CRISPRi) system using plas- 
mids pKLH116 (pNZ8148 containing an inducible promoter $\mathrm{P}_{\text {nisin }}$ to drive the expression of dCas9 from Streptococcus pyogenes, Supplemental Figure S1; https://doi .org/10.3168/jds.2019-17346) and pSGRNAs (pNZ44 containing a strong constitutive promoter $\mathrm{P}_{44}$ to drive expression of single or multiple sgRNAs, Supplemental Figure S2; https://doi.org/10.3168/jds.2019-17346) in L. lactis NZ9000. All strains, primers, and methods used in this study are presented in Supplemental File S1 and Supplemental Tables S1 and S2 (https://doi .org/10.3168/jds.2019-17346). The dCas9 in pKLH116 was replaced by the reporter red fluorescence protein (Supplemental Figure S3; https://doi.org/10.3168/jds .2019-17346) to assess the optimal nisin (the inducer) concentration for protein expression. The fluorescence intensity of red fluorescence protein indicated that the optimal concentration of nisin is $35 \mu \mathrm{g} / \mathrm{L}$ for recombinant protein expression. At this concentration of nisin, dCas9 was obviously expressed (Supplemental Figure S4; https://doi.org/10.3168/jds.2019-17346), but the growth of cells expressing dCas9, sgRNA, or dCas9 and sgRNA was not significantly changed (Supplemental Figure S5; https://doi.org/10.3168/jds.2019-17346). These results suggest that the CRISPRi system does not impair cell viability.

An overview of our CRISPRi system is shown in Figure 1. To demonstrate the efficiency of this system, we experimentally repressed the expression of 3 genes in NZ9000: upp (encoding uracil phosphoribosyltransferase), sod (encoding superoxide dismutase), and LLNZ_07335 (encoding putative penicillin acylase in the protein annotation of NZ9000 genome). The CRISPRi system is highly sensitive to mismatches between sgRNA and its target site, and it has minimal off-target effects if the sgRNA is carefully designed (Radzisheuskaya et al., 2016). Hence, we designed sgRNAs targeted to 6 different positions of upp, sod, and $L L N Z \_07335$ (Supplemental Figure S2; https://doi.org/10.3168/jds .2019-17346), respectively. All sgRNAs significantly repressed the expression of target genes from 31 to $98 \%$, suggesting that sgRNA targeting at any position of a gene sequence has the potential for efficient gene interference (Figure 2). Uracil phosphoribosyltransferase is capable of transforming 5-fluorouracil (5-FU) to uridine monophosphate, which inhibits the activity of thymidylate synthase. Consequently, 5-FU is fatal to cells with high-level expression of upp. Conversely, deletion or knockdown of upp makes bacteria resistant to 5-FU (Song et al., 2017). Compared with the control without sgRNA, upp expression was significantly decreased by 70 to $98 \%$ (Figure 2A). Cells without sgRNA targeting upp cannot grow on semi-defined medium (SDM) containing $50 \mathrm{mg} / \mathrm{L}$ of 5-FU (SDMF50), but cells with sgRNAs targeting upp can grow on SDMF50 due to knockdown of upp expression (Figure 2B). This result was accordance with a previous report (Berlec et al., 2018) showing that upp expression could be knocked down by dCas9. The expression of sod was also repressed by 48 to $80 \%$ with sgRNAs targeting sod (Figure 2C), resulting in a 27 to $57 \%$ decrease in enzyme activity (Figure 2D). The results suggest that this CRISPRi system can be used to efficiently repress a single gene in L. lactis.

Bile salt hydrolase (BSH), which catalyzes the release of free amino acids from bile salts (Xiong et al., 2017), is responsible for bile salt resistance in LAB. Although L. lactis exhibits bile salt tolerance, BSH has not been previously reported in L. lactis. Based on sequence alignment and similarity analysis of NZ9000 genome using the known BSHs from Lactobacillus (Xiong et al., 2017) as the references, LLNZ_07335, a putative protein, was mined that could be attributed to the family of BSH in L. lactis (see Supplemental Figure S6; https://doi.org/10.3168/jds.2019-17346). Phylogenetic analysis showed that LLNZ_07335 had the highest sequence identity (59\%) with BSH of Lactobacillus casei Zhang (Supplemental Figure S6; https://doi.org/ 10.3168/jds.2019-17346). Therefore, sgRNAs targeting LLNZ_07335 were designed to test whether gene repression can affect the bile salt resistance of NZ9000. Compared with the control without sgRNA, the expression of LLNZ_07335 in cells harboring sgRNA showed a 1.5- to 5.1-fold decrease (Figure 2E), resulting in 9.3 to $67.2 \%$ bacterial survival on GM17 medium containing $0.04 \%$ bovine bile (Figure $2 \mathrm{~F}$ ). This result indicates that LLNZ_07335 is a protein of the BSH family enabling bile salt resistance. The most important advantage of gene knockdown over gene knockout is that it can be used to study essential genes (Peters et al., 2016; Zhao et al., 2017). Hence, the CRISPRi system used here offers the means for systematic investigation of essential gene functions in L. lactis. Moreover, due to the small size of plasmid pSGRNAs (3,170 bp) in this system, it is possible to rapidly construct a sgRNA library for high-throughput screening of functional genes by using a simple inverse PCR method (Larson et al., 2013).

Whether our CRISPRi system can simultaneously repress multiple target genes was explored in L. lactis. The plasmid pSGRNA was used to carry multiple sgRNA expression cassettes (Supplemental Figure S2; https://doi.org/10.3168/jds.2019-17346). The sgRNAs $u p p$-sgUF and LLNZ_07335-sgBD were expressed separately or together in NZ9000. When expressed alone, each sgRNA specifically repressed its target gene but did not affect the expression of the other gene (Figure $3 \mathrm{~A})$. When upp-sgUF and $L L N Z \_07335$-sgBD were 


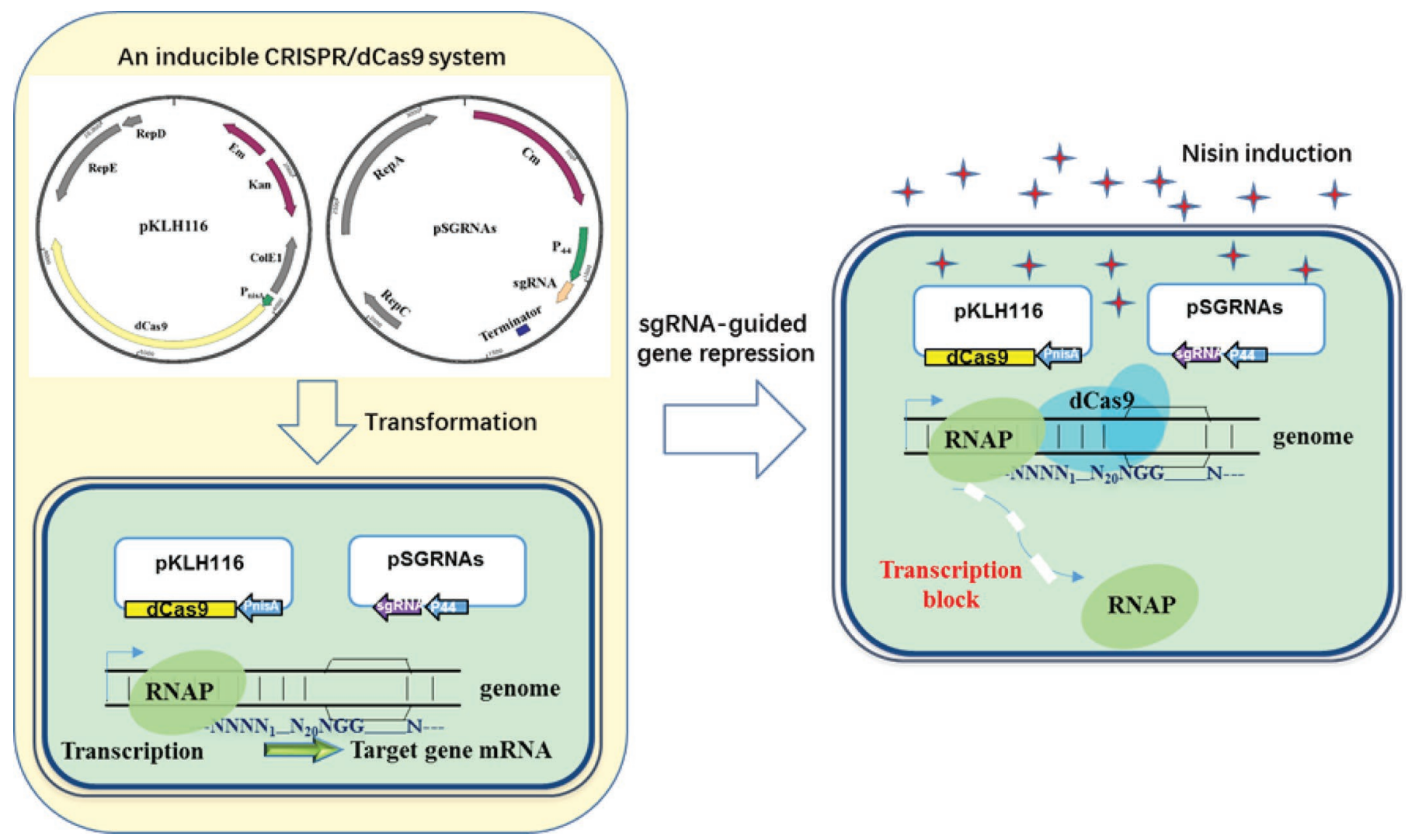

Figure 1. Overview of the clustered regularly interspaced short palindromic repeats (CRISPR) interference gene repression system in Lactobacillus lactis. Step 1 is co-transformation of pKLH116 and pSGRNA into L. lactis. In step 2, with the addition of the inducer nisin, single guide (sg)RNA-guided dCas9 (nuclease-inactivated Cas9 or dead Cas9) binding to genomic DNA generates a complex that produces a certain steric hindrance that represses the transcription of target gene. RNAP $=$ RNA polymerase.

expressed together, upp and LLNZ_07335 were significantly repressed at a lower rate than when either sgRNA was expressed alone (Figure 3A). This finding could be attributed to the competitive binding of dCas9 by the 2 sgRNAs (Zhao et al., 2017). The expression of 2 sgRNAs both targeting upp also enhanced the effect of knockdown (Figure 3B). The results suggest that this system can be used to specifically repress multiple genes simultaneously in L. lactis.

The CRISPRi system is a precise method for genome regulation by gene silencing. Silencing is achieved by using sgRNA-guided dCas9 to target specific genes or promoters, which in turn physically inhibits mRNA transcription and elongation (Larson et al., 2013). In this work, an inducible CRISPRi system for gene knockdown was developed in L. lactis. This CRISPRi-mediated silencing of single or multiple genes significantly repressed gene expression up to $99 \%$. Compared with the findings of Berlec et al. (2018), our results improved the use of dCas9 for gene knockdown from a single gene to multiple genes in L. lactis. Moreover, we identified BSH in L. lactis by using this CRISPRi system. To our knowledge, our report is the first for functional gene identification in LAB by using the CRISPRi method. Gene knockdown technology has been widely applied in gene function studies and microbial metabolic engineering (Na et al., 2013), and our CRISPRi system could be a valuable supplement to the genetic toolbox for gene silencing in LAB. With all evidence taken together, this gene repression tool has the potential for screening of functional genes and applications of metabolic engineering and synthetic biology in LAB.

\section{ACKNOWLEDGMENTS}

This work was supported by the National Key R\&D Program of China (grant No. 2018YFD0502306), National Natural Science Foundation of China (grant no. 31871776 and 31771956), Natural Science Foundation of Shanghai (grant no. 18ZR1426800), Shanghai Agriculture Applied Technology Development Program (grant no. 2019-02-08-00-07-F01152), and Shanghai Engineering Research Center of Food Microbiology (grant no. 19DZ2281100). 
A

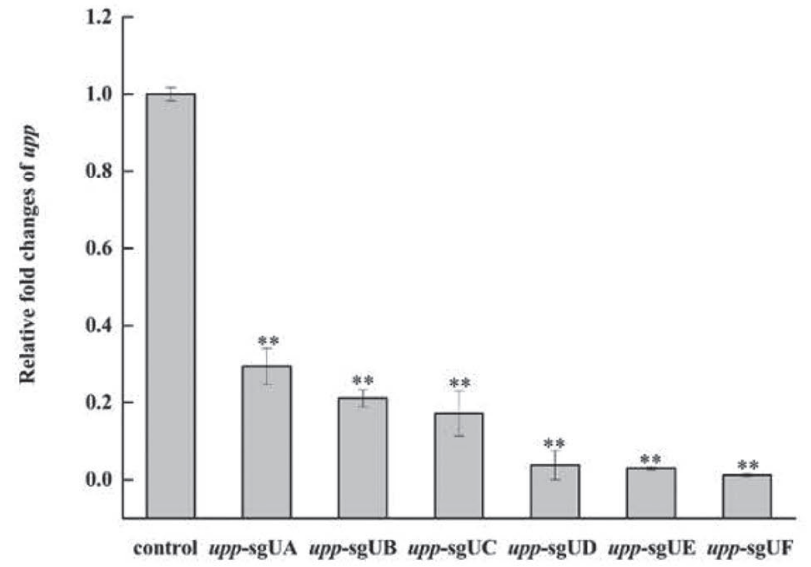

C

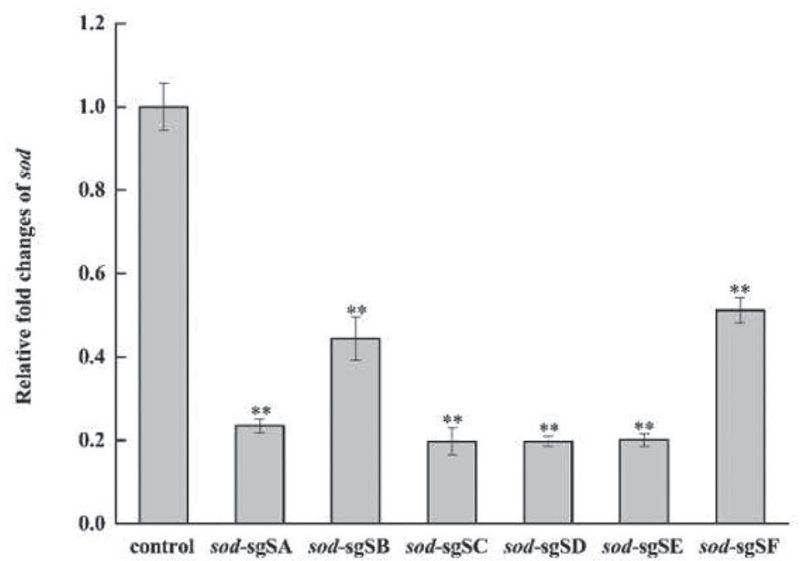

$E$

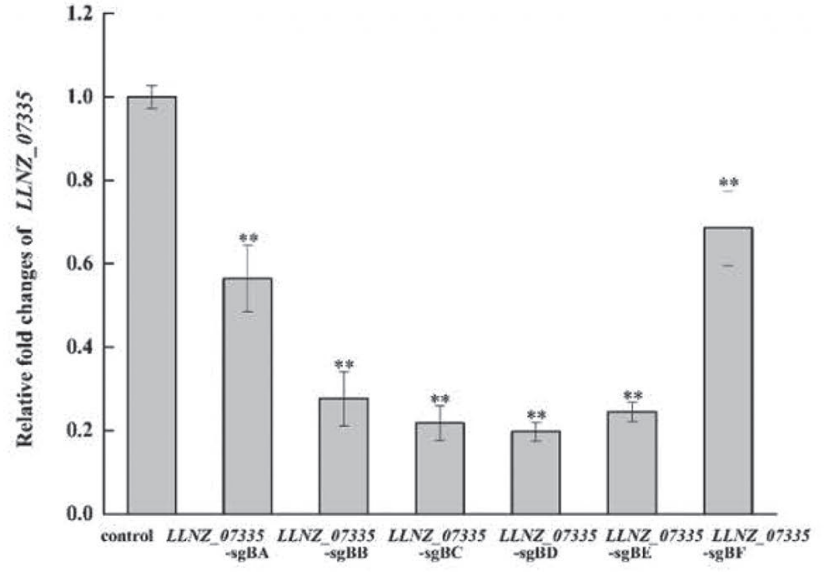

B

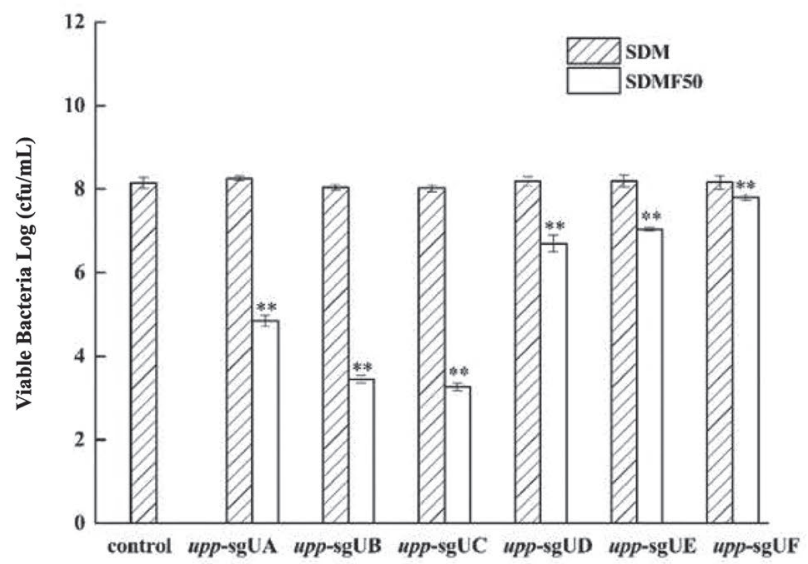

D

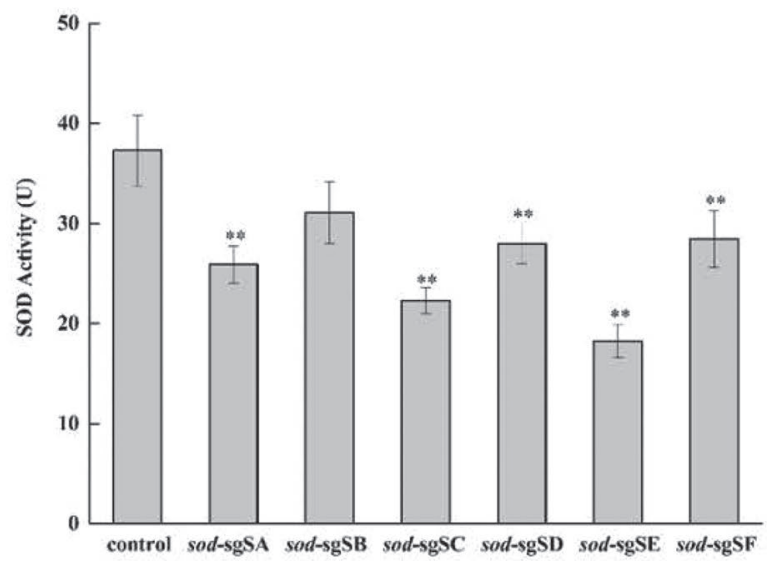

$\mathbf{F}$

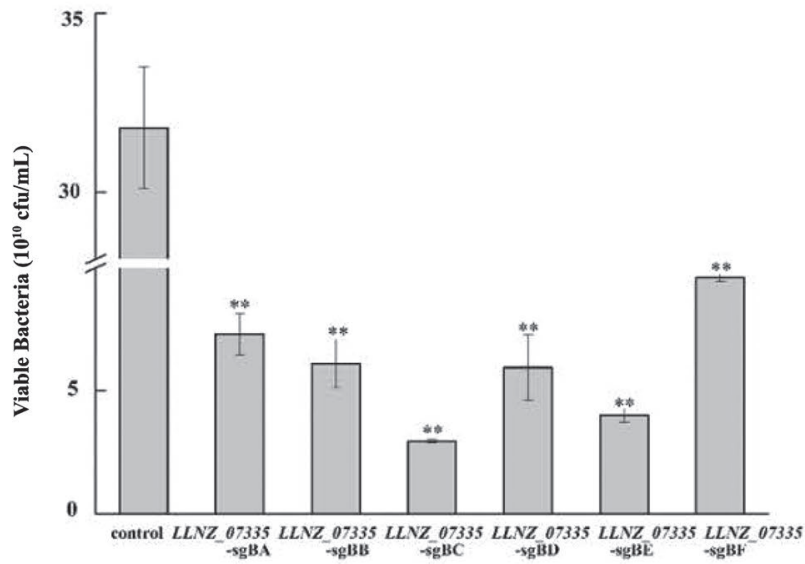

Figure 2. Gene knockdown using the clustered regularly interspaced short palindromic repeats (CRISPR) interference system with single guide (sg)RNA in Lactobacillus lactis. (A) Normalized transcription level of upp (encoding uracil phosphoribosyltransferase) using different sgRNA. (B) Survival of L. lactis transformed with different sgRNA targeting upp in semi-defined medium (SDM) and in SDM with 50 mg/L 5-fluorouracil (SDMF50). (C) Normalized transcription level of sod (encoding superoxide dismutase) using different sgRNA. (D) Superoxide dismutase (SOD) activity of strains harboring different sgRNA targeting sod. (E) Normalized transcription level of $L L N Z \_07335$ (encoding putative penicillin acylase in the protein annotation of NZ9000 genome) using different sgRNA. (F) Survival in $0.04 \%$ bovine bile of $L$. lactis transformed with different sgRNA of $L L N Z \_07335$ gene. ${ }^{* *} P<0.01$ vs. control group. Error bars indicate SD of the mean of triplicates. 
A

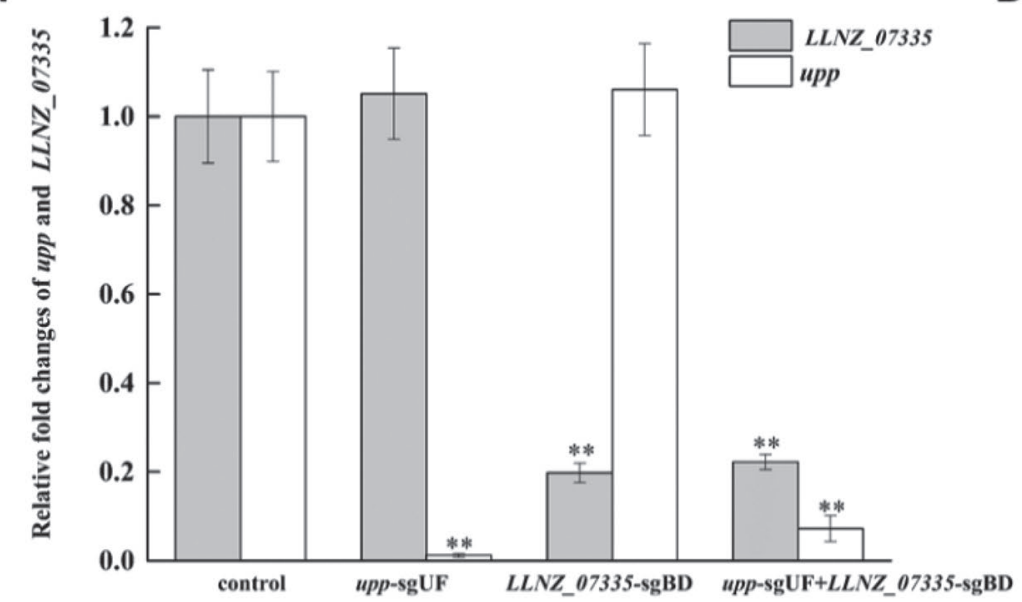

B

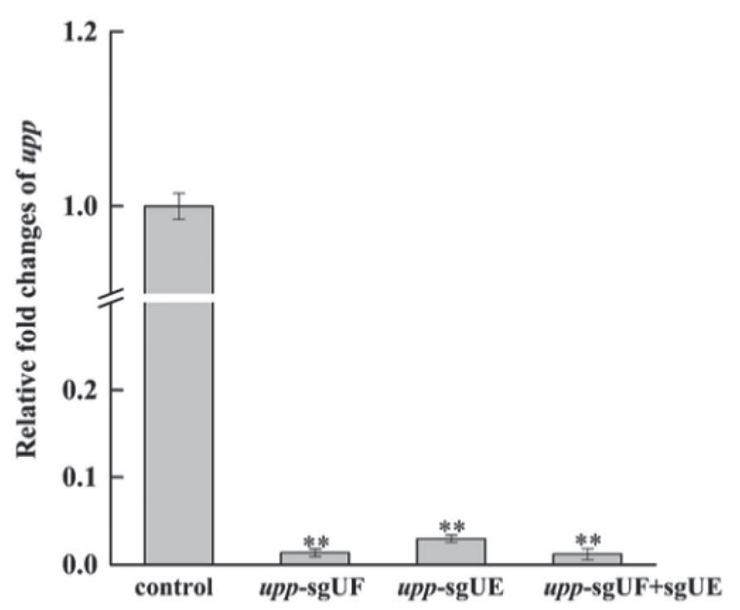

Figure 3. Gene knockdown using the clustered regularly interspaced short palindromic repeats (CRISPR) interference system with multiple single guide (sg)RNA in Lactobacillus lactis. (A) Normalized transcription level of upp (encoding uracil phosphoribosyltransferase) and LLNZ_07335 (encoding putative penicillin acylase in the protein annotation of NZ9000 genome) using sgRNA. (B) Normalized transcription level of upp using 2 different sgRNA for multiple repression. ${ }^{* *} P<0.01$ vs. control group. Error bars indicate SD of the mean of triplicates.

\section{REFERENCES}

Berlec, A., K. Skrlec, J. Kocjan, M. Olenic, and B. Strukelj. 2018. Single plasmid systems for inducible dual protein expression and for CRISPR-Cas9/CRISPRi gene regulation in lactic acid bacterium Lactococcus lactis. Sci. Rep. 8:1009.

Hidalgo-Cantabrana, C., S. O'Flaherty, and R. Barrangou. 2017. CRISPR-based engineering of next-generation lactic acid bacteria. Curr. Opin. Microbiol. 37:79-87.

Huang, H., X. Song, and S. Yang. 2019. Development of a RecE/Tassisted CRISPR-Cas9 toolbox for Lactobacillus. Biotechnol. J. 14:e1800690.

Larson, M. H., L. A. Gilbert, X. Wang, W. A. Lim, J. S. Weissman, and L. S. Qi. 2013. CRISPR interference (CRISPRi) for sequencespecific control of gene expression. Nat. Protoc. 8:2180-2196.

Na, D., S. M. Yoo, H. Chung, H. Park, J. H. Park, and S. Y. Lee. 2013. Metabolic engineering of Escherichia coli using synthetic small regulatory RNAs. Nat. Biotechnol. 31:170-174.

Oh, J. H., and J. P. van Pijkeren. 2014. CRISPR-Cas9-assisted recombineering in Lactobacillus reuteri. Nucleic Acids Res. 42:e131.

Peters, J. M., A. Colavin, H. Shi, T. L. Czarny, M. H. Larson, S. Wong, J. S. Hawkins, C. H. S. Lu, B. M. Koo, E. Marta, A. L. Shiver, E. H. Whitehead, J. S. Weissman, E. D. Brown, L. S. Qi, K. C. Huang, and C. A. Gross. 2016. A comprehensive, CRIS-
PR-based functional analysis of essential genes in bacteria. Cell 165:1493-1506.

Radzisheuskaya, A., D. Shlyueva, I. Muller, and K. Helin. 2016. Optimizing sgRNA position markedly improves the efficiency of CRISPR/dCas9-mediated transcriptional repression. Nucleic Acids Res. 44:e141.

Song, X., H. Huang, Z. Xiong, L. Ai, and S. Yang. 2017. CRISPRCas9 ${ }^{\mathrm{D} 10 \mathrm{~A}}$ nickase-assisted genome editing in Lactobacillus casei Appl. Environ. Microbiol. 83:e01259-17.

Xiong, Z. Q., Q. H. Wang, L. H. Kong, X. Song, G. Q. Wang, Y. J. Xia, H. Zhang, Y. Sun, and L. Z. Ai. 2017. Short communication: Improving the activity of bile salt hydrolases in Lactobacillus casei based on in silico molecular docking and heterologous expression. J. Dairy Sci. 100:975-980.

Zhao, C., X. Shu, and B. Sun. 2017. Construction of a gene knockdown system based on catalytically inactive ("dead") Cas9 (dCas9) in Staphylococcus aureus. Appl. Environ. Microbiol. 83:e0291-17.

\section{ORCIDS}

Zhi-Qiang Xiong (ํ) https://orcid.org/0000-0002-7984-3662

Hua-Xi Yi ৫ https://orcid.org/0000-0002-8968-4263

Lian-Zhong Ai @ https://orcid.org/0000-0002-6681-9102 